\title{
The exact theory for scattering of waves by thick holes in a slab and other objects with non-separable geometries
}

\section{B. J. Hoenders \\ b.j.hoenders@rug.nl}

\section{University of Groningen, Center for Theoretical Physics Zernike Institute for Advanced Materials, Nijenborgh 4, NL-9747 AG Groningen, The Netherlands}

The theory for scattering of electromagnetic waves is developed for scattering objects for which the natural modes of the field inside the object do not couple one-to-one with those outside the scatterer. Key feature of the calculation of the scattered fields is the introduction of a new set of modes. As an example, we calculate the reflected and transmitted fields generated by an electromagnetic plane wave that impinges upon a multilayer slab of which the layers are stacked perpendicular to the boundary planes. As this is the geometry of a thick plate with slits our theory encompasses the exact scattering theory of electromagnetic waves by a thick plate with slits. [DOI: 10.2971/jeos.2011.11011s]

Keywords: exact scattering theory, non separable geometries, thick holes

\section{INTRODUCTION}

The analysis of scattering- and boundary value problems is a very important branch of physics and it has been extensively explored since the eighteenth century $[1,2]$. One of the requisites for the possibility to obtain analytical solutions in closed form for these problems is that the pertinent scalar- or vectorial wave equation admits a potential or refractive index such that this equation separates. Another requirement, essential for the analytical solution of scattering- and boundary value problems, is that the geometry of the scatterer fits with the geometry of the separable potential or refractive index. In the case of scattering from, for instance, a sphere or cylinder, the potential or index of refraction should have a spherical or cylindrical dependence, i.e. the level surfaces of the potential or refractive index must coincide with the boundary surfaces. Then, the field is calculated employing the technique of eigenfunction expansions which are generated by the set of ordinary differential equations that result from the separation of the original partial differential equation, viz. the scalar- or vectorial wave equation [1]-[4].

The exactly solvable boundary value problems in mathematical physics all share one property, namely that the boundaries of the various geometries involved fully coincide with the coordinate surfaces of the various separable coordinate systems for the wave equation. This is the case for the scattering of waves from, for instance, a half-plane, a complete sphere, an ellipsoid or a cylinder filled with a homogeneous medium. The boundaries of all these objects fully coincide with a separable coordinate system. However, no simple theory exists for the calculation of fields that have been scattered from media with so-called non-separable boundaries. For the scattering from, for example, a wedge or a half-sphere, the theory becomes much more difficult and no simple theory exists if these objects consist of materials with respectively a layered structure or a radially dependent refractive index. No exact theory exists in the sense given above for the scattering from, for instance, an insect eye or an array of rectangular protrusions (telegrapher's surface), see Figure 1. We would also like to mention the configuration drawn in Figure 3. This figure shows a so-called rotated multilayer. Each layer has finite width and length. Moreover, a special case of the geometry drawn in Figure 3 is that of several slits in a thick plate. This statement is immediately understood if we make the special choices $\varepsilon_{L}, \mu_{L}=\varepsilon_{R}, \mu_{R}=\varepsilon_{A}, \mu_{A}$ for the pertinent material parameters.

Because the solutions of such scattering problems are not knownin terms of a series of eigenfunctions with known coefficients they are called: Not exactly solvable problems. The absence of a simple theory for scattering from objects with nonseparable boundaries stems from the following observation: one natural mode of the field outside the scattering medium no longer couples to one natural mode inside the scatterer but

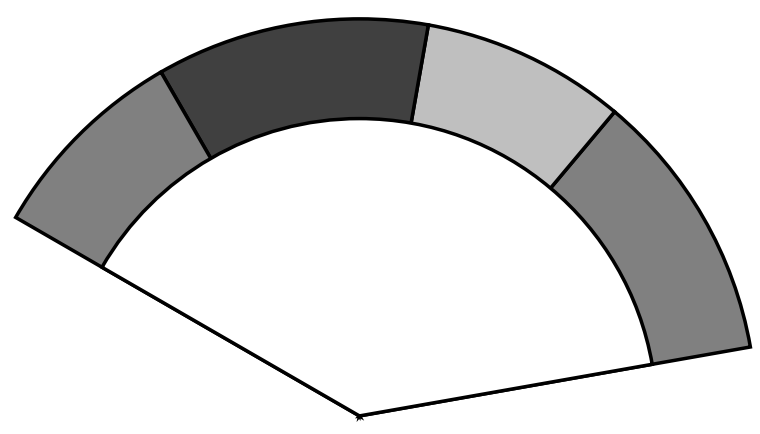

FIG. 1a Model examples of objects in which there is no one-to-one coupling of the internal and external field modes: the two-dimensional insect eye. Different colors indicate regions in which the refractive index can take on different values. The light is supposed to enter from above. 


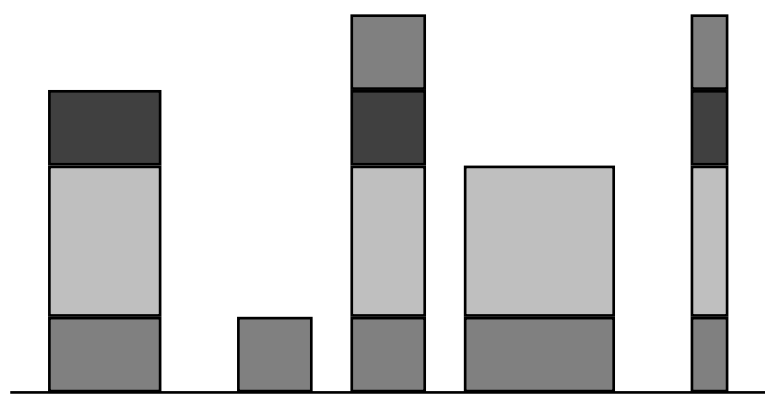

FIG. 1b Model examples of objects in which there is no one-to-one coupling of the internal and external field modes: the telegrapher surface. Different colors indicate regions in which the refractive index can take on different values. The light is supposed to enter from above.

to an, in general, infinite number of modes. This observation is corroborated analyzing the results of the Wiener-Hopf technique [5], which is, for instance, used for the description of scattering of waves by a half-cylinder and connects an infinity of modes outside and inside the scatterer. As an example that supports this statement, one could think of a slab that is made from layers with different constant indices of refraction such that the layers are perpendicular to the planes of the slab. Then, a plane wave mode outside the slab couples to an infinity of modes for the layered medium, unlike for the case if the layers had been oriented parallel to the boundary planes of the slab. In the latter case, one plane wave mode outside couples to one plane wave mode inside the multilayer.

This paper shows, however, that with the introduction of new, different, sets of modes (one set for the inside- and one set for the outside of the scattering medium), it is again as if one outside mode couples to one inside mode, which is one of the essential requirements for exactly solvable scattering problems. This property of the new set of modes thus enables one to solve the scattering problem the same way as for the usual exactly solvable problems. In particular, we will consider the scattering of an electromagnetic wave by a multilayer slab, (see Figure 3) such that the layers are oriented perpendicular to the boundary planes of the slab, viz. the layers are rotated over 90 degrees with respect to the orientation considered in the ordinary theory of layered media. As mentioned before, a particular case of this geometry is that of several slits in a thick plate. A survey of the background theory of the mathematical results that have been used in this chapter and can be found in [6], whereas the original theory in full detail can be found in [7].

\section{HYBRID MODE EXPANSIONS}

In this section, the general theory for the scattering of TEor TM-polarized electromagnetic waves from a slab of which the response to the field varies along the boundary surface is treated.

Figure illustrates the type of medium we have in mind. In following sections, specific examples of such media will be treated. In the absence of free electric charges and currents,

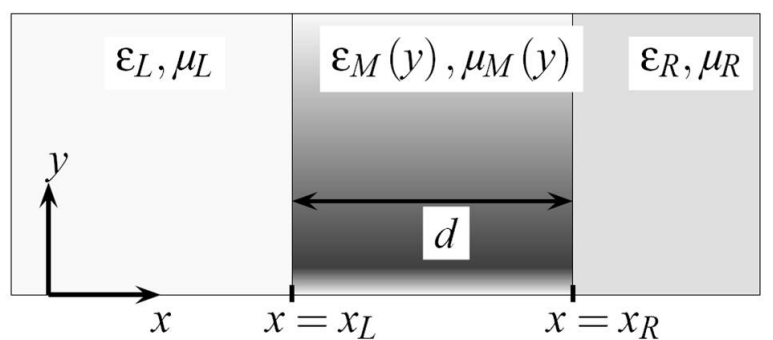

FIG. 2 A slab of which the permittivity $\varepsilon_{M}$ and permeability $\mu_{M}$ vary along the boundary surface. The electromagnetic waves are supposed to scatter from the slab sideedges at $x=x_{L}$ and $x=x_{R}$. The width of the slab is $d$.

Maxwell's equations read as

$$
\begin{aligned}
\nabla \times \mathbf{E}+\dot{\mathbf{B}} & =0, \\
\nabla \times \mathbf{H}-\dot{\mathbf{D}} & =0, \\
\nabla \cdot \mathbf{D} & =0, \\
\nabla \cdot \mathbf{B} & =0,
\end{aligned}
$$

where $\mathbf{E}$ is the electric field, $\mathbf{B}$ the magnetic induction, $\mathbf{D}$ the displacement field and $\mathbf{H}$ the magnetic field. Let $\mathbf{G}$ denote one of these fields. In Fourier representation,

$$
\begin{aligned}
\mathbf{G}(t, \mathbf{r}) & =\int \mathrm{d} \omega \widetilde{\mathbf{G}}(\omega ; \mathbf{r}) \exp (-\mathrm{i} \omega t), \\
\text { where } \widetilde{\mathbf{G}}(\omega ; \mathbf{r}) & =\frac{1}{2 \pi} \int \mathrm{d} t \mathbf{G}(t, \mathbf{r}) \exp (\mathrm{i} \omega t) .
\end{aligned}
$$

For linear and isotropic media,

$$
\begin{aligned}
\widetilde{\mathbf{D}} & =\varepsilon \widetilde{\mathbf{E}}, \\
\widetilde{\mathbf{H}} & =\mu^{-1} \widetilde{\mathbf{B}},
\end{aligned}
$$

where $\varepsilon$ and $\mu$ are respectively the absolute permittivity and permeability, Eqs. (1) give

$$
\begin{aligned}
\left(\nabla^{2}+\omega^{2} \varepsilon \mu\right) \widetilde{\mathbf{E}}+(\nabla \ln \mu) & \times \nabla \\
& \times \widetilde{\mathbf{E}} \\
\left(\nabla^{2}+\omega^{2} \varepsilon \mu\right) \widetilde{\mathbf{H}}+(\nabla \ln \varepsilon) & \times \nabla \times \widetilde{\operatorname{H}} \varepsilon)=0 \\
& +\nabla(\widetilde{\mathbf{H}} \cdot \nabla \ln \mu)=0 .
\end{aligned}
$$

Denote

$$
\begin{gathered}
\eta^{E}=\varepsilon, \\
\eta^{H}=\mu .
\end{gathered}
$$

The media to the left- and to the right of the slab are homogeneous and, inside the slab, the response functions vary across the boundary planes of the slab, hence

$$
\eta^{F}(x, y)=\left\{\begin{array}{ll}
\eta_{L^{\prime}}^{F}, & x<x_{L}, \\
\eta_{M}^{F}(y), & x_{L}<x<x_{R}, \\
\eta_{R^{\prime}}^{F} & x>x_{R}
\end{array} \quad F=E, H,\right.
$$

see Figure 2. There is no variation in the response functions in the $z$ direction, which is the direction "outside the plane of the paper". For TE-polarization, $\mathbf{E}=E \hat{\mathbf{z}}$ and the magnetic field follows from Eq. (1a) as

$$
\widetilde{\mathbf{H}}=\frac{\mathrm{i}}{\omega \mu}\left(-\hat{\mathbf{x}} \partial_{y} \widetilde{E}+\hat{\mathbf{y}} \partial_{x} \widetilde{E}\right) .
$$


For TM-polarization, $\mathbf{H}=H \hat{\mathbf{z}}$ and the electric field follows from Eq. (1b) as

$$
\widetilde{\mathbf{E}}=\frac{\mathrm{i}}{\omega \varepsilon}\left(\hat{\mathbf{x}} \partial_{y} \widetilde{H}-\hat{\mathbf{y}} \partial_{x} \widetilde{H}\right) .
$$

We will only consider either TE- or TM-polarization. Let $F$ denote the amplitude of the z-component of the field, i.e. $F=E$ in case of TE-polarization and $F=H$ in case of TMpolarization. In the various regions of space, the field is labeled in accordance with the response functions of Eq. (6) as

$$
F=\left\{\begin{array}{l}
F_{L}, \quad x<x_{L} \\
F_{M}, \quad x_{L}<x<x_{R} \\
F_{R,} \quad x>x_{R} .
\end{array}\right.
$$

Eqs. (4) give that $(m=L, R)$

$$
\begin{array}{r}
{\left[\partial_{x}^{2}+\partial_{y}^{2}+k_{m}^{2}\right] \widetilde{F}_{m}=0,} \\
{\left[\partial_{x}^{2}+\partial_{y}^{2}+\omega^{2} \varepsilon_{M} \mu_{M}-\left(\partial_{y} \ln \bar{\eta}_{M}^{F}\right) \partial_{y}\right] \widetilde{F}_{M}=0}
\end{array}
$$

where

$$
k_{m}^{2}=\omega^{2} \varepsilon_{m} \mu_{m}
$$

and where we introduced

$$
\begin{gathered}
\bar{\eta}^{E}=\mu, \\
\bar{\eta}^{H}=\varepsilon .
\end{gathered}
$$

For Eqs. (10), mode solutions of the following forms are tried:

$$
\begin{aligned}
& \widetilde{F}_{m}\left(f^{2} ; x, y\right)=\phi_{m}\left(f^{2} ; x\right) \psi_{m}\left(f^{2} ; y\right), \\
& \widetilde{F}_{M}\left(f^{2} ; x, y\right)=\phi_{M}\left(f^{2} ; x\right) \psi_{M}\left(f^{2} ; y\right) .
\end{aligned}
$$

Of these, the trial modes outside the slab are indeed solutions if

$$
\begin{aligned}
& \left(\partial_{x}^{2}+f_{m}^{2}\right) \phi_{m}\left(f^{2} ; x\right)=0 \\
& \left(\partial_{y}^{2}+f^{2}\right) \psi_{m}\left(f^{2} ; y\right)=0
\end{aligned}
$$

where $f^{2}$ is the separation constant and where

$$
f_{m}^{2}=k_{m}^{2}-f^{2} .
$$

The trial modes inside the slab are solutions if they satisfy

$$
\begin{aligned}
& \left(\partial_{x}^{2}+f_{L}^{2}\right) \phi_{M}\left(f^{2} ; x\right)=0, \\
& \left(\partial_{y}^{2}+f^{2}\right) \psi_{M}\left(f^{2} ; y\right)=K_{M}^{F}\left(f^{2} ; y\right),
\end{aligned}
$$

where

$$
K_{M}^{F}=\left(k_{L}^{2}-\omega^{2} \varepsilon_{M} \mu_{M}\right) \psi_{M}+\left(\partial_{y} \ln \bar{\eta}_{M}^{F}\right) \partial_{y} \psi_{M} .
$$

Our specific choice for $K_{M}^{F}$ implies that, when media $L$ and $M$ are chosen equal, i.e. when there is no scattering at the interface at $x=x_{L}$, this corresponds to having $K_{M}^{F}=0$. In App. 7 it is shown that the functions $\psi_{m}$ and $\psi_{M}$ satisfy the following completeness relation

$$
\delta\left(y-y^{\prime}\right)=\sum_{n} \frac{\psi_{m}\left(f_{n}^{2} ; y\right) \psi_{M}\left(f_{n}^{2} ; y^{\prime}\right)}{N^{\prime}\left(f_{n}^{2}\right)},
$$

where the sum is over those separation constants $f_{n}^{2}$ that satisfy Eq. (98), and where $N^{\prime}\left(f_{n}^{2}\right)=\left.\left(\mathrm{d} N / \mathrm{d} f^{2}\right)\right|_{f^{2}=f_{n}^{2}}$. and $N^{\prime}\left(f_{n}^{2}\right)$ is defined by Eq. (98) of the appendix. The completeness relation Eq. (18) gives an expansion for the deltadistribution in terms of the free space modes and the medium modes, therefore we call it the hybrid mode expansion.

The rightwards (r)- and leftwards (l) propagating field solutions to Eqs. (14) are, in the pertinent mode expansions, in the various parts of space, given by

$$
\begin{aligned}
\widetilde{F}_{m}^{(\mathrm{r} / \mathrm{l})}(x, y)= & \sum_{n} \bar{\rho}_{m}^{(\mathrm{r} / \mathrm{l})}\left(f_{n}^{2}\right) \\
& \times \exp \left( \pm \mathrm{i} f_{n, m}\left(x-x_{m}\right)\right) \psi_{m}\left(f_{n}^{2} ; y\right), \\
\widetilde{F}_{M}^{(\mathrm{r} / \mathrm{l})}(x, y)= & \sum_{n} \rho_{M}^{(\mathrm{r} / \mathrm{l})}\left(f_{n}^{2}\right) \\
& \times \exp \left( \pm \mathrm{i} f_{n, L}\left(x-x_{L}\right)\right) \psi_{M}\left(f_{n}^{2} ; y\right),
\end{aligned}
$$

where the plus-(minus-)signs belong to the rightwards (leftwards) propagating fields and where

$$
f_{n, m}^{2}=k_{m}^{2}-f_{n}^{2} .
$$

From Eqs. (18) it follows that the spectral densities in Eqs. (19) are given by

$$
\begin{aligned}
\bar{\rho}_{m}^{(\mathrm{r} / \mathrm{l})}\left(f_{n}^{2}\right)= & \left(N^{\prime}\left(f_{n}^{2}\right)\right)^{-1} \\
& \times \int_{0}^{1} \mathrm{~d} y^{\prime} \widetilde{F}_{m}^{(\mathrm{r} / \mathrm{l})}\left(x_{m}, y^{\prime}\right) \bar{\psi}_{m}\left(f_{n}^{2} ; y^{\prime}\right), \\
\rho_{M}^{(\mathrm{r} / \mathrm{l})}\left(f_{n}^{2}\right)= & \left(N^{\prime}\left(f_{n}^{2}\right)\right)^{-1} \\
& \times \int_{0}^{1} \mathrm{~d} y^{\prime} \widetilde{F}_{M}^{(\mathrm{r} / \mathrm{l})}\left(x_{L}, y^{\prime}\right) \psi_{m}\left(f_{n}^{2} ; y^{\prime}\right) .
\end{aligned}
$$

The unknown spectral densities $\bar{\rho}_{L}^{(1)}, \rho_{M}^{(\mathrm{r} / 1)}$ and $\bar{\rho}_{R}^{(\mathrm{r})}$ of the four scattered fields are determined from the conditions of continuity of the tangential components of the electric and magnetic fields at the two boundaries,

$$
\begin{aligned}
\left.\left(\widetilde{F}_{L}^{(1)}+\widetilde{F}_{L}^{(\mathrm{r})}\right)\right|_{x} & =x_{L} \\
& =\left.\left(\widetilde{F}_{M}^{(1)}+\widetilde{F}_{M}^{(\mathrm{r})}\right)\right|_{x=x_{L}}{ }^{\prime} \\
\left.\frac{1}{\bar{\eta}_{L}^{F}}\left(\partial_{x} \widetilde{F}_{L}^{(1)}+\partial_{x} \widetilde{F}_{L}^{(\mathrm{r})}\right)\right|_{x} & =x_{L} \\
& =\left.\frac{1}{\bar{\eta}_{M}^{F}}\left(\partial_{x} \widetilde{F}_{M}^{(1)}+\partial_{x} \widetilde{F}_{M}^{(\mathrm{r})}\right)\right|_{x=x_{L}}, \\
& =\left.\left(\widetilde{F}_{R}^{(1)}+\widetilde{F}_{R}^{(\mathrm{r})}\right)\right|_{x=x_{R}}{ }^{\prime} \\
\left.\frac{1}{\bar{\eta}_{M}^{F}}\left(\partial_{x} \widetilde{F}_{M}^{(1)}+\partial_{x} \widetilde{F}_{M}^{(\mathrm{r})}\right)\right|_{x} & =x_{R} \\
& =\left.\frac{1}{\bar{\eta}_{R}^{F}}\left(\partial_{x} \widetilde{F}_{R}^{(1)}+\partial_{x} \widetilde{F}_{R}^{(\mathrm{r})}\right)\right|_{x=x_{R}} .
\end{aligned}
$$

Eqs. (22b) and (22d) were obtained from Eqs. (7) and (8). The conditions of Eq. (22) lead to a set of four coupled linear integral equations for the unknown densities.

The conditions of Eq. (22) do not lead to a simple system of equations, because, as follows from inserting the fields of 
Eq. (19), both types of modes $\psi_{m}$ and $\psi_{M}$ are involved. It is only in the case that these modes are identical ${ }^{1}$, that the spectral densities can be equated for each mode. But, this much desired property is obtained from the hybrid mode expansion of Eq. (18), which allows for the following rewriting of the mode expansions for the fields inside the slab. Starting from Eq. (19b), with Eq. (21b), we have that

$$
\begin{aligned}
\widetilde{F}_{M}^{(\mathrm{r} / \mathrm{l})}(x, y)= & \sum_{n}\left(N^{\prime}\left(f_{n}^{2}\right)\right)^{-1} \\
& \times \int_{0}^{1} \mathrm{~d} y^{\prime} \widetilde{F}_{M}^{(\mathrm{r} / \mathrm{l})}\left(x_{L}, y^{\prime}\right) \psi_{m}\left(f_{n}^{2} ; y^{\prime}\right) \\
& \times \exp \left( \pm \mathrm{i} f_{n, L}\left(x-x_{L}\right)\right) \psi_{M}\left(f_{n}^{2} ; y\right) .
\end{aligned}
$$

From Eq. (20), the differential equation for $\psi_{M}$ (Eq. (16b)) and (17) it follows that

$$
\begin{aligned}
f_{n, L} \psi_{M} & \left(f_{n}^{2} ; y\right) \\
= & \sqrt{\partial_{y}^{2}+\varepsilon_{M}(y) \mu_{M}(y) \omega^{2}-\left(\partial_{y} \ln \bar{\eta}_{M}^{F}(y)\right) \partial_{y}} \\
& \times \psi_{M}\left(f_{n}^{2} ; y\right) .
\end{aligned}
$$

Hence, Eq. (23) can be written as

$$
\begin{aligned}
\widetilde{F}_{M}^{(\mathrm{r} / 1)} & (x, y)=\exp \left( \pm \mathrm{i}\left(x-x_{L}\right)\right. \\
& \left.\times \sqrt{\partial_{y}^{2}+\varepsilon_{M}(y) \mu_{M}(y) \omega^{2}-\left(\partial_{y} \ln \bar{\eta}_{M}^{F}(y)\right) \partial_{y}}\right) \\
& \times \int_{0}^{1} \mathrm{~d} y^{\prime} \widetilde{F}_{M}^{(\mathrm{r} / 1)}\left(x_{L}, y^{\prime}\right) \sum_{n}\left(N^{\prime}\left(f_{n}^{2}\right)\right)^{-1} \\
& \times \psi_{m}\left(f_{n}^{2} ; y^{\prime}\right) \psi_{M}\left(f_{n}^{2} ; y\right) .
\end{aligned}
$$

From the hybrid mode expansion of Eq. (18), which is symmetric in $y$ and $y^{\prime}$, it follows that Eq. (25) must be equal to

$$
\begin{aligned}
\widetilde{F}_{M}^{(\mathrm{r} / \mathrm{l})} & (x, y)=\exp \left( \pm \mathrm{i}\left(x-x_{L}\right)\right. \\
& \left.\times \sqrt{\partial_{y}^{2}+\varepsilon_{M}(y) \mu_{M}(y) \omega^{2}-\left(\partial_{y} \ln \bar{\eta}_{M}^{F}(y)\right) \partial_{y}}\right) \\
& \times \int_{0}^{1} \mathrm{~d} y^{\prime} \widetilde{F}_{M}^{(\mathrm{r} / 1)}\left(x_{L}, y^{\prime}\right) \sum_{n}\left(N^{\prime}\left(f_{n}^{2}\right)\right)^{-1} \\
& \times \psi_{M}\left(f_{n}^{2} ; y^{\prime}\right) \psi_{m}\left(f_{n}^{2} ; y\right) .
\end{aligned}
$$

Observe the symmetrical change of the arguments of the functions and $\psi_{m}$ and $\psi_{m}$ in resp. Eqs. (25) and (26)! Now, the derivatives act on $\psi_{m}$ and from using the pertinent differential equation, Eq. (14b), it follows that Eq. (26) equals

$$
\begin{aligned}
\widetilde{F}_{M}^{(\mathrm{r} / \mathrm{l})}(x, y)= & \sum_{n} \sigma_{M}^{(\mathrm{r} / \mathrm{l})}\left(f_{n}^{2}\right) \\
& \times \exp \left( \pm \mathrm{i} f_{n, M}(y)\left(x-x_{L}\right)\right) \psi_{m}\left(f_{n}^{2} ; y\right),
\end{aligned}
$$

where

$$
f_{n, M}(y)=\sqrt{\varepsilon_{M}(y) \mu_{M}(y) \omega^{2}-\mathrm{i} f_{n}\left(\partial_{y} \ln \bar{\eta}_{M}^{F}(y)\right)-f_{n}^{2}}
$$

\footnotetext{
slab.

${ }^{1}$ Recall that this is the case for instance the scattering from a homogeneous
}

and with now

$$
\begin{aligned}
\sigma_{M}^{(\mathrm{r} / \mathrm{l})}\left(f_{n}^{2}\right)= & \left(N^{\prime}\left(f_{n}^{2}\right)\right)^{-1} \\
& \times \int_{0}^{1} \mathrm{~d} y^{\prime} \widetilde{F}_{M}^{(\mathrm{r} / 1)}\left(x_{L}, y^{\prime}\right) \psi_{M}\left(f_{n}^{2} ; y^{\prime}\right) .
\end{aligned}
$$

It can be verified that, in the representation of Eq. (27), $\widetilde{F}_{M}^{(\mathrm{r} / 1)}$ satisfies Eq. (10b). The conditions of Eq. (22) are then applied to the fields of Eqs. (19a) and (27). Equating the coefficients of the modes $\left\{\psi_{m}\left(f_{n}^{2} ; y\right)\right\}$ at both sides of the interface at $x=x_{L}$ leads to ${ }^{2}$

$$
\Delta_{L}\left(f_{n}^{2}\right)\left(\begin{array}{ll}
\bar{\rho}_{L}^{(\mathrm{r})}\left(f_{n}^{2}\right) \\
\bar{\rho}_{L}^{(1)} & \left(f_{n}^{2}\right)
\end{array}\right)=\Delta_{M}\left(f_{n}^{2} ; y\right)\left(\begin{array}{c}
\sigma_{M}^{(\mathrm{r})}\left(f_{n}^{2}\right) \\
\sigma_{M}^{(1)}\left(f_{n}^{2}\right)
\end{array}\right)
$$

where the dynamical matrices are given by

$$
\begin{aligned}
\Delta_{m}\left(f^{2}\right) & =\left(\begin{array}{cc}
1 & 1 \\
\frac{f_{m}}{\bar{\eta}_{m}^{F}} & -\frac{f_{m}}{\bar{\eta}_{m}^{F}}
\end{array}\right), \\
\Delta_{M}\left(f^{2} ; y\right) & =\left(\begin{array}{cc}
1 & 1 \\
\frac{f_{M}(y)}{\bar{\eta}_{M}^{F}(y)} & -\frac{f_{M}(y)}{\bar{\eta}_{M}^{F}(y)}
\end{array}\right) .
\end{aligned}
$$

Equating the coefficients of the modes $\left\{\psi_{m}\left(f_{n}^{2} ; y^{\prime}\right)\right\}$ at both sides of the interface at $x=x_{R}$ gives

$$
\begin{aligned}
& \Delta_{M}\left(f_{n}^{2} ; y^{\prime}\right) P_{M}\left(f_{n}^{2} ; y^{\prime}\right)\left(\begin{array}{ll}
\sigma_{M}^{(\mathrm{r})}\left(f_{n}^{2}\right) \\
\sigma_{M}^{(1)}\left(f_{n}^{2}\right)
\end{array}\right) \\
& =\Delta_{R}\left(f_{n}^{2}\right)\left(\begin{array}{ll}
\bar{\rho}_{R}^{(\mathrm{r})} & \left(f_{n}^{2}\right) \\
\bar{\rho}_{R}^{(1)} & \left(f_{n}^{2}\right)
\end{array}\right),
\end{aligned}
$$

where $d=x_{R}-x_{L}$ and where the propagation matrix is given by

$$
P_{M}\left(f^{2} ; y\right)=\operatorname{diag}\left(\exp \left(\mathrm{i} f_{M}(y) d\right), \exp \left(-\mathrm{i} f_{M}(y) d\right)\right)
$$

Eqs. (30) and (33) can be solved for the unknown spectral densities of the scattered fields using Cramer's rule. Thus, one finds the inhomogeneous equivalents of the Fresnel transmission and reflection coefficients for the interface between homogeneous medium $L$ and inhomogeneous medium $M$ respectively as

$$
\begin{aligned}
t_{L M}\left(f_{n}^{2} ; y\right) & \left.\equiv\left(\frac{\sigma_{M}^{(\mathrm{r})}\left(f_{n}^{2}\right)}{\bar{\rho}_{L}^{(\mathrm{r})}\left(f_{n}^{2}\right)}\right)\right|_{\sigma_{M}^{(1)}\left(f_{n}^{2}\right)=0} \\
& =\frac{2\left(f_{n, L} / \bar{\eta}_{L}^{F}\right)}{\left(f_{n, L} / \bar{\eta}_{L}^{F}\right)+\left(f_{n, M}(y) / \bar{\eta}_{M}^{F}(y)\right)}, \\
r_{L M}\left(f_{n}^{2} ; y\right) & \left.\equiv\left(\frac{\bar{\rho}_{L}^{(1)}\left(f_{n}^{2}\right)}{\bar{\rho}_{L}^{(\mathrm{r})}\left(f_{n}^{2}\right)}\right)\right|_{\sigma_{M}^{(1)}\left(f_{n}^{2}\right)=0} \\
& =\frac{\left(f_{n, L} / \bar{\eta}_{L}^{F}\right)-\left(f_{n, M}(y) / \bar{\eta}_{M}^{F}(y)\right)}{\left(f_{n, L} / \bar{\eta}_{L}^{F}\right)+\left(f_{n, M}(y) / \bar{\eta}_{M}^{F}(y)\right)},
\end{aligned}
$$

Similar coefficients are obtained for the interface at $x=x_{R}$. This gives the $y$-dependent transmission and reflection coefficients of the complete slab for the rightwards propagating

\footnotetext{
${ }^{2}$ Note that the boundary conditions of Eqs. (30) and (33) reduce to the boundary conditions for a homogeneous slab when $\varepsilon_{M}$ and $\mu_{M}$ do not depend on $y$.
} 
waves of Eq. (19a) respectively as

$$
\begin{aligned}
& t_{M}\left(f_{n}^{2} ; y\right) \\
& \equiv\left.\left(\frac{\bar{\rho}_{R}^{(\mathrm{r})}\left(f_{n}^{2}\right)}{\bar{\rho}_{L}^{(\mathrm{r})}\left(f_{n}^{2}\right)}\right)\right|_{\rho_{R}^{(1)}\left(f_{n}^{2}\right)=0} \\
&= \frac{t_{L M}\left(f_{n}^{2} ; y\right) t_{M R}\left(f_{n}^{2} ; y\right) \exp \left(\mathrm{i} f_{n, M}(y) d\right)}{1-r_{M L}\left(f_{n}^{2} ; y\right) r_{M R}\left(f_{n}^{2} ; y\right) \exp \left(2 \mathrm{i} f_{n, M}(y) d\right)}, \\
& r_{M}\left(f_{n}^{2} ; y\right) \\
& \equiv \\
&=\left.\left(\frac{\bar{\rho}_{L}^{(1)}\left(f_{n}^{2}\right)}{\bar{\rho}_{L}^{(\mathrm{r})}\left(f_{n}^{2}\right)}\right)\right|_{\rho_{R}^{(1)}\left(f_{n}^{2}\right)=0} \\
& \quad r_{L M}\left(f_{n}^{2} ; y\right) \\
& \quad+\frac{t_{L M}\left(f_{n}^{2} ; y\right) t_{M L}\left(f_{n}^{2} ; y\right)}{1} \\
& \quad \times \frac{r_{M R}\left(f_{n}^{2} ; y\right) \exp \left(2 \mathrm{i} f_{n, M}(y) d\right)}{1-r_{M L}\left(f_{n}^{2} ; y\right) r_{M R}\left(f_{n}^{2} ; y\right) \exp \left(2 \mathrm{i} f_{n, M}(y) d\right)} .
\end{aligned}
$$

The reflected and transmitted fields that result from a field $F_{L}^{(\mathrm{r})}$ that is applied from the left to the slab follow respectively as

$$
\begin{aligned}
\widetilde{F}_{L}^{(1)}(x, y)= & \sum_{n} r_{M}\left(f_{n}^{2} ; y\right) \bar{\rho}_{L}^{(\mathrm{r})}\left(f_{n}^{2}\right) \\
& \times \exp \left(-\mathrm{i} f_{n, L}\left(x-x_{L}\right)\right) \psi_{m}\left(f_{n}^{2} ; y\right), \\
\widetilde{F}_{R}^{(\mathrm{r})}(x, y)= & \sum_{n} t_{M}\left(f_{n}^{2} ; y\right) \bar{\rho}_{L}^{(\mathrm{r})}\left(f_{n}^{2}\right) \\
& \times \exp \left(\mathrm{i} f_{n, R}\left(x-x_{R}\right)\right) \psi_{m}\left(f_{n}^{2} ; y\right) .
\end{aligned}
$$

\section{MODES IN THE ROTATED MULTILAYER SLAB}

As a specific example of the scattering geometry that has been depicted in Figure 2, we consider a multilayer which consists of $N$ periods of alternating slabs $\sigma=A, B$ with thicknesses $l_{\sigma}$, permittivities $\varepsilon_{\sigma}$ and permeabilities $\mu_{\sigma}$. The geometry is depicted in Figure 3. The electromagnetic field is supposed to enter the medium through its boundaries at $x=x_{L}$, or/and $x=x_{R}$. Therefore, as compared to its conventional orientation with respect to the applied field, the multilayer of Figure 3 is rotated over 90 degrees in the $x y$-plane. The right- and leftwards propagating parts of the field inside the medium are given, in their expansions in the free space modes, by Eq. (27),

$$
\begin{aligned}
\widetilde{F}_{M}^{(\mathrm{r} / \mathrm{l})}(x, y)= & \sum_{n} \sigma_{M}^{(\mathrm{r} / \mathrm{l})}\left(f_{n}^{2}\right) \\
& \times \exp \left( \pm \mathrm{i} f_{n, M}(y)\left(x-x_{L}\right)\right) \psi_{m}\left(f_{n}^{2} ; y\right),
\end{aligned}
$$

where the spectral densities are given by

$$
\begin{aligned}
\sigma_{M}^{(\mathrm{r} / \mathrm{l})}\left(f_{n}^{2}\right)= & \left(N^{\prime}\left(f_{n}^{2}\right)\right)^{-1} \\
& \times \int_{0}^{1} \mathrm{~d} y^{\prime} \widetilde{F}_{M}^{(\mathrm{r} / 1)}\left(x_{L}, y^{\prime}\right) \psi_{M}\left(f_{n}^{2} ; y^{\prime}\right),
\end{aligned}
$$

and where

$$
f_{n, M}(y)=f_{n, \sigma} \quad \text { if } \quad y_{\sigma \lambda} \leq y<y_{\sigma \lambda}+l_{\sigma \lambda}
$$

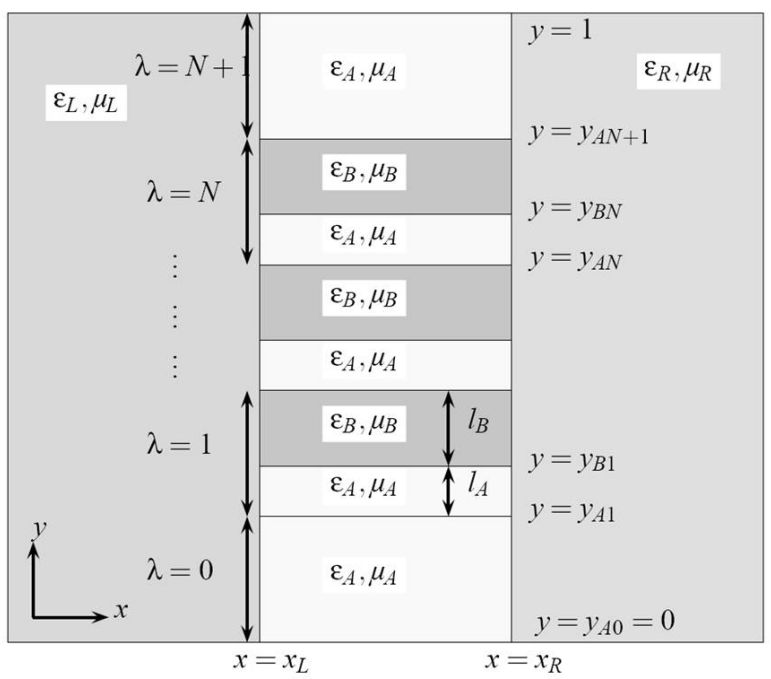

FIG. 3 The rotated multilayer. The width, permittivity and permeability of slab $\sigma=A, B$ are respectively $l_{\sigma}, \varepsilon_{\sigma}$ and $\mu_{\sigma}$ and the $y_{\sigma \lambda}$ denote the coordinates of the interfaces between the slabs.

with $y_{\sigma \lambda}$ the interface coordinates, see Figure 3 . We further defined

$$
l_{A 0}=y_{A 1}, l_{A N+1}=1-y_{A N+1}
$$

and

$$
l_{\sigma \lambda}=l_{\sigma} \text { if } \lambda=1, \ldots, N
$$

The modes inside the medium, for

$$
y_{\sigma \lambda} \leq y \leq y_{\sigma \lambda}+l_{\sigma \lambda}
$$

are given by

$$
\begin{aligned}
\psi_{M}\left(f^{2} ; y\right)= & \chi_{\sigma \lambda}^{(u)} \exp \left(\mathrm{i} f_{\sigma L}\left(y-y_{\sigma \lambda}\right)\right) \\
& +\chi_{\sigma \lambda}^{(d)} \exp \left(-\mathrm{i} f_{\sigma L}\left(y-y_{\sigma \lambda}\right)\right),
\end{aligned}
$$

with $f_{\sigma L}=\sqrt{k_{\sigma}^{2}-f_{L}^{2}}$. The coefficients $\chi_{\sigma \lambda}^{(u)}$ and $\chi_{\sigma \lambda}^{(d)}$ of respectively the up- and downwards propagating parts of the modes in Eq. (41) satisfy [8]

$$
\begin{aligned}
& \left(\begin{array}{l}
\chi_{B 1}^{(u)} \\
\chi_{B 1}^{(d)}
\end{array}\right)=\Theta_{B A} P_{A}\left(\begin{array}{l}
\chi_{A 1}^{(u)} \\
\chi_{A 1}^{(d)}
\end{array}\right) \\
& \left(\begin{array}{l}
\chi_{\sigma \lambda}^{(u)} \\
\chi_{\sigma \lambda}^{(d)}
\end{array}\right)=\left(\frac{\varepsilon^{\lambda-1}-\varepsilon^{1-\lambda}}{\varepsilon-\varepsilon^{-1}} T_{\sigma}-\frac{\varepsilon^{\lambda-2}-\varepsilon^{2-\lambda}}{\varepsilon-\varepsilon^{-1}}\right)\left(\begin{array}{c}
\chi_{\sigma 1}^{(u)} \\
\chi_{\sigma 1}^{(d)}
\end{array}\right),
\end{aligned}
$$

where the transmission and propagation matrices are respectively given by

$$
\begin{aligned}
\Theta_{B A} & =\frac{1}{2}\left(\begin{array}{ll}
1+\eta_{A B} & 1-\eta_{A B} \\
1-\eta_{A B} & 1+\eta_{A B}
\end{array}\right), \quad \eta_{A B} \equiv \frac{\bar{\eta}_{B}}{\bar{\eta}_{A}} \frac{f_{A L}}{f_{B L}}, \\
P_{\sigma} & =\operatorname{diag}\left(\exp \left(\mathrm{i} f_{\sigma L} l_{\sigma}\right), \exp \left(-\mathrm{i} f_{\sigma L} l_{\sigma}\right)\right),
\end{aligned}
$$

and where $\varepsilon=\frac{1}{2} \operatorname{tr} T_{\sigma}+\sqrt{\left(\frac{1}{2} \operatorname{tr} T_{\sigma}\right)^{2}-1}$ and $\varepsilon^{-1}$ are the eigenvalues of the single-layer transfer matrix,

$$
T_{\sigma}=\left(\begin{array}{cc}
A_{\sigma} & B_{\sigma} \\
C_{\sigma} & D_{\sigma}
\end{array}\right)
$$


for transfer between successive slabs $\sigma$. The entries of this matrix are given by $[9,10]$

$$
\begin{aligned}
& A_{\sigma}=\exp \left(\mathrm{i} f_{\sigma L} l_{\sigma}\right) \\
& \times\left[\cos \left(f_{\bar{\sigma} L} l_{\bar{\sigma}}\right)+\frac{\mathrm{i}}{2}\left(\frac{\bar{\eta}_{\sigma}^{F}}{\bar{\eta}_{\bar{\sigma}}^{F}} \frac{f_{\bar{\sigma} L}}{f_{\sigma L}}+\frac{\bar{\eta}_{\bar{\sigma}}^{F}}{\bar{\eta}_{\sigma}^{F}} \frac{f_{\sigma L}}{f_{\bar{\sigma} L}}\right) \sin \left(f_{\bar{\sigma} L} l_{\bar{\sigma}}\right)\right], \\
& B_{\sigma}=\frac{\mathrm{i}}{2} \exp \left(-\mathrm{i} f_{\sigma L} l_{\sigma}\right)\left(\frac{\bar{\eta}_{\sigma}^{F}}{\bar{\eta}_{\bar{\sigma}}^{F}} \frac{f_{\bar{\sigma} L}}{f_{\sigma L}}-\frac{\bar{\eta}_{\bar{\sigma}}^{F}}{\bar{\eta}_{\sigma}^{F}} \frac{f_{\sigma L}}{f_{\bar{\sigma} L}}\right) \sin \left(f_{\bar{\sigma} L} l_{\bar{\sigma}}\right), \\
& C_{\sigma}=\frac{-\mathrm{i}}{2} \exp \left(\mathrm{i} f_{\sigma L} l_{\sigma}\right)\left(\frac{\bar{\eta}_{\sigma}^{F}}{\bar{\eta}_{\bar{\sigma}}^{F}} \frac{f_{\bar{\sigma} L}}{f_{\sigma L}}-\frac{\bar{\eta}_{\bar{\sigma}}^{F}}{\bar{\eta}_{\sigma}^{F}} \frac{f_{\sigma L}}{f_{\bar{\sigma} L}}\right) \sin \left(f_{\bar{\sigma} L} l_{\bar{\sigma}}\right), \\
& D_{\sigma}=\exp \left(-\mathrm{i} f_{\sigma L} l_{\sigma}\right) \\
& \times\left[\cos \left(f_{\bar{\sigma} L} l_{\bar{\sigma}}\right)-\frac{\mathrm{i}}{2}\left(\frac{\bar{\eta}_{\sigma}^{F}}{\bar{\eta}_{\bar{\sigma}}^{F}} \frac{f_{\bar{\sigma} L}}{f_{\sigma L}}+\frac{\bar{\eta}_{\bar{\sigma}}^{F}}{\bar{\eta}_{\sigma}^{F}} \frac{f_{\sigma L}}{f_{\bar{\sigma} L}}\right) \sin \left(f_{\bar{\sigma} L} l_{\bar{\sigma}}\right)\right],
\end{aligned}
$$

where $\bar{\sigma}=B$ if $\sigma=A$ and $\bar{\sigma}=A$ if $\sigma=B$. The modes outside and inside the medium fulfil Eq. (18) if they satisfy the boundary conditions

$$
\begin{aligned}
\psi_{m}\left(f^{2} ; 1\right) & =h_{1}, \\
\left.\left(\partial_{y} \psi_{m}\left(f^{2} ; y\right)\right)\right|_{y=1} & =h_{2},
\end{aligned}
$$

and

$$
\begin{array}{r}
\psi_{M}\left(f^{2} ; 0\right)=\alpha, \\
\left.\left(\partial_{y} \psi_{M}\left(f^{2} ; y\right)\right)\right|_{y=0}=\beta,
\end{array}
$$

and if the separation constant $f^{2}$ satisfies the discretisation condition $N\left(f^{2}\right)=0$, with $N$ from Eq. (98). This condition implies

$$
\left.h_{1}\left(\partial_{y} \psi_{M}\left(f_{n}^{2} ; y\right)\right)\right|_{y=1}-h_{2} \psi_{M}\left(f_{n}^{2} ; 1\right)=\gamma .
$$

The appropriate set of modes $\left\{\psi_{M}\left(f_{n}^{2} ; y\right)\right\}$ has now been defined for the rotated multilayer, so that the scattering theory developed above can be applied to this particular system.

\section{SCATTERING FROM A LAYER WITH FINITE WIDTH}

In this last section, we calculate the fields that are scattered from an interface between a homogeneous medium and a medium with one layer with two slabs that have finite widths. The geometry has been depicted in Figure 4 . As before, the incoming $\left(F_{L}^{(\mathrm{r})}\right)$, reflected $\left(F_{L}^{(1)}\right)$ and transmitted $\left(F_{M}^{(\mathrm{r})}\right)$ fields are expressed in their mode expansions,

$$
\begin{gathered}
\widetilde{F}_{L}^{(\mathrm{r} / \mathrm{l})}(x, y)=\sum_{n} \rho_{L}^{(\mathrm{r} / \mathrm{l})}\left(f_{n}^{2}\right) \exp \left( \pm \mathrm{i} f_{n, L} x\right) \psi_{L}\left(f_{n}^{2} ; y\right), \\
\widetilde{F}_{M}^{(\mathrm{r})}(x, y)=\sum_{n} \rho_{M}^{(\mathrm{r})}\left(f_{n}^{2}\right) \exp \left(\mathrm{i} f_{n, L} x\right) \psi_{M}\left(f_{n}^{2} ; y\right),
\end{gathered}
$$

where

$$
\begin{aligned}
\rho_{L}^{(\mathrm{r} / \mathrm{l})}\left(f_{n}^{2}\right)= & \left(N^{\prime}\left(f_{n}^{2}\right)\right)^{-1} \\
& \times \int_{0}^{1} \mathrm{~d} y^{\prime} \widetilde{F}_{L}^{(\mathrm{r} / \mathrm{l})}\left(0, y^{\prime}\right) \psi_{L}\left(f_{n}^{2} ; y^{\prime}\right), \\
\rho_{M}^{(r)}\left(f_{n}^{2}\right)= & \left(N^{\prime}\left(f_{n}^{2}\right)\right)^{-1} \\
& \times \int_{0}^{1} \mathrm{~d} y^{\prime} \widetilde{F}_{M}^{(r)}\left(0, y^{\prime}\right) \psi_{L}\left(f_{n}^{2} ; y^{\prime}\right) .
\end{aligned}
$$

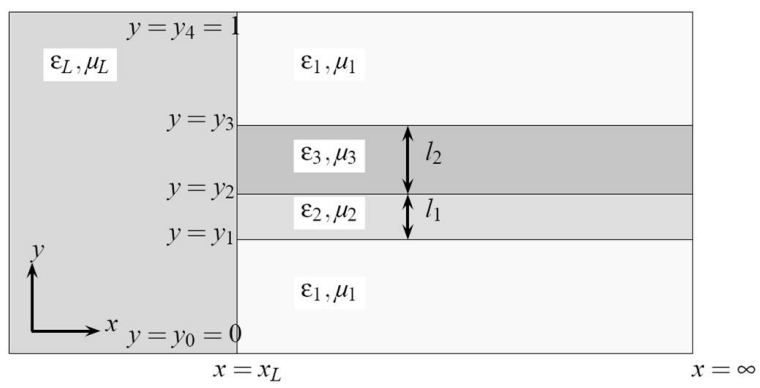

FIG. 4 Geometry used for the numerical calculation.

Continuity of the tangential components of the electric and magnetic fields at $x=0$ gives for the various fields $F$ that

$$
\begin{gathered}
\left.\left(\widetilde{F}_{L}^{(\mathrm{r})}+\widetilde{F}_{L}^{(1)}\right)\right|_{x=0}=\left.\widetilde{F}_{M}^{(\mathrm{r})}\right|_{x=0}{ }^{\prime} \\
\left.\frac{1}{\bar{\eta}_{L}^{F}}\left(\partial_{x}\left(\widetilde{F}_{L}^{(\mathrm{r})}+\widetilde{F}_{L}^{(\mathrm{l})}\right)\right)\right|_{x=0}=\left.\frac{1}{\bar{\eta}_{R}^{F}}\left(\partial_{x} \widetilde{F}_{M}^{(\mathrm{r})}\right)\right|_{x=0}
\end{gathered}
$$

Eq. (52b) implies, with Eqs. (50), that

$$
\begin{array}{r}
\left(1 / \bar{\eta}_{L}^{F}\right) \sum_{n} f_{n, L}\left(\rho_{L}^{(\mathrm{r})}\left(f_{n}^{2}\right)-\rho_{L}^{(1)}\left(f_{n}^{2}\right)\right) \psi_{L}\left(f_{n}^{2} ; y\right) \\
=\left(1 / \bar{\eta}_{M}^{F}\right) \sum_{n} f_{n, L} \rho_{M}^{(\mathrm{r})}\left(f_{n}^{2}\right) \psi_{M}\left(f_{n}^{2} ; y\right) .
\end{array}
$$

The left- and right hand side of Eq. (53) are series expansions into the set of modes $\left\{\psi_{L}\left(f_{n}^{2} ; y\right)\right\}$ and $\left\{\psi_{M}\left(f_{n}^{2} ; y\right)\right\}$ resp.. We would like to express both sides of Eq. (53) on the same basis, say $\psi_{M}$. Hereto, first expand the mode densities. For the lefthand-side of Eq. (53), this gives

$$
\begin{gathered}
\left(1 / \bar{\eta}_{L}^{F}\right) \sum_{n} f_{n, L}\left(\rho_{L}^{(\mathrm{r})}\left(f_{n}^{2}\right)-\rho_{L}^{(1)}\left(f_{n}^{2}\right)\right) \psi_{L}\left(f_{n}^{2} ; y\right) \\
=\left(1 / \bar{\eta}_{L}^{F}\right) \int \mathrm{d} y^{\prime}\left(\widetilde{F}_{L}^{(\mathrm{r})}\left(0, y^{\prime}\right)-\widetilde{F}_{L}^{(1)}\left(0, y^{\prime}\right)\right) \\
\quad \times \sum_{n} \frac{\psi_{L}\left(f_{n}^{2} ; y\right) f_{n, L} \psi_{M}\left(f_{n}^{2} ; y^{\prime}\right)}{N^{\prime}\left(f_{n}^{2}\right)}
\end{gathered}
$$

and for the right-hand-side of Eq. (53),

$$
\begin{aligned}
&\left(1 / \bar{\eta}_{M}^{F}\right) \sum_{n} f_{n, L} \rho_{M}^{(\mathrm{r})}\left(f_{n}^{2}\right) \psi_{M}\left(f_{n}^{2} ; y\right) \\
&=\left(1 / \bar{\eta}_{M}^{F}\right) \int \mathrm{d} y^{\prime} \widetilde{F}_{M}^{(\mathrm{r})}\left(0, y^{\prime}\right) \\
& \times \sum_{n} \frac{\psi_{L}\left(f_{n}^{2} ; y^{\prime}\right) f_{n, L} \psi_{M}\left(f_{n}^{2} ; y\right)}{N^{\prime}\left(f_{n}^{2}\right)} .
\end{aligned}
$$

Then, note that Eqs. (15), (16b) and (17) imply that

$$
\begin{aligned}
& f_{n, L} \psi_{M}\left(f_{n}^{2} ; y^{\prime}\right) \\
& =\sqrt{\partial_{y^{\prime}}^{2}+\omega^{2} \varepsilon_{M}\left(y^{\prime}\right) \mu_{M}\left(y^{\prime}\right)-\left(\partial_{y^{\prime}} \ln \bar{\eta}_{M}^{F}\left(y^{\prime}\right)\right) \partial_{y^{\prime}}} \\
& \quad \times \psi_{M}\left(f_{n}^{2} ; y^{\prime}\right) .
\end{aligned}
$$

The completeness relation Eq. (18) implies

$$
\sum_{n} \frac{\psi_{L}\left(f_{n}^{2} ; y^{\prime}\right) \psi_{M}\left(f_{n}^{2} ; y\right)}{N^{\prime}\left(f_{n}^{2}\right)}=\sum_{n} \frac{\psi_{L}\left(f_{n}^{2} ; y\right) \psi_{M}\left(f_{n}^{2} ; y^{\prime}\right)}{N^{\prime}\left(f_{n}^{2}\right)} \text {. }
$$


The square root operator in Eq. (56) can thus be switched to act on $\psi_{L}$ instead of $\psi_{M}$. With using Eq. (14b), it follows that

$$
\begin{aligned}
& \sqrt{\partial_{y^{\prime}}^{2}+\omega^{2} \varepsilon_{M}\left(y^{\prime}\right) \mu_{M}\left(y^{\prime}\right)-\left(\partial_{y^{\prime}} \ln \bar{\eta}_{M}^{F}\left(y^{\prime}\right)\right) \partial_{y^{\prime}}} \psi_{L}\left(f_{n}^{2} ; y^{\prime}\right) \\
& =\sqrt{\omega^{2} \varepsilon_{M}\left(y^{\prime}\right) \mu_{M}\left(y^{\prime}\right)-\mathrm{i} f_{n}\left(\partial_{y^{\prime}} \ln \bar{\eta}_{M}^{F}\left(y^{\prime}\right)\right)-f_{n}^{2}} \\
& \quad \times \psi_{L}\left(f_{n}^{2} ; y^{\prime}\right) .
\end{aligned}
$$

Equating coefficients of $\psi_{M}$ gives thus that a solution to Eq. (53) is given by

$$
\begin{aligned}
\left(1 / \bar{\eta}_{L}^{F}\right) \int \mathrm{d} y^{\prime}\left(\widetilde{F}_{L}^{(\mathrm{r})}\left(0, y^{\prime}\right)-\widetilde{F}_{L}^{(1)}\left(0, y^{\prime}\right)\right) \\
\times \sqrt{\omega^{2} \varepsilon_{M}\left(y^{\prime}\right) \mu_{M}\left(y^{\prime}\right)-\mathrm{i} f_{n}\left(\partial_{y^{\prime}} \ln \bar{\eta}_{M}^{F}\left(y^{\prime}\right)\right)-f_{n}^{2}} \\
\quad \times \psi_{L}\left(f_{n}^{2} ; y^{\prime}\right) \\
=\left(f_{n, L} / \bar{\eta}_{M}^{F}(y)\right) \int \mathrm{d} y^{\prime} \widetilde{F}_{M}^{(\mathrm{r})}\left(0, y^{\prime}\right) \psi_{L}\left(f_{n}^{2} ; y^{\prime}\right) .
\end{aligned}
$$

Since this holds for all $f_{n}^{2}$, it must hold for all $f^{2}$. With Eq. (52a), this gives that the reflected field is related to the applied field as

$$
\begin{aligned}
& \int \mathrm{d} y^{\prime} \widetilde{F}_{L}^{(1)}\left(0, y^{\prime}\right) \psi_{L}\left(f^{2} ; y^{\prime}\right) \times\left(\left(1 / \bar{\eta}_{L}^{F}\right)\right. \\
& \times \sqrt{\omega^{2} \varepsilon_{M}\left(y^{\prime}\right) \mu_{M}\left(y^{\prime}\right)-\mathrm{i} f\left(\partial_{y^{\prime}} \ln \bar{\eta}_{M}^{F}\left(y^{\prime}\right)\right)-f^{2}} \\
& \left.\quad+\left(f_{L} / \bar{\eta}_{M}^{F}(y)\right)\right) \\
& =\int \mathrm{d} y^{\prime} \widetilde{F}_{L}^{(\mathrm{r})}\left(0, y^{\prime}\right) \psi_{L}\left(f^{2} ; y^{\prime}\right) \times\left(\left(1 / \bar{\eta}_{L}^{F}\right)\right. \\
& \quad \times \sqrt{\omega^{2} \varepsilon_{M}\left(y^{\prime}\right) \mu_{M}\left(y^{\prime}\right)-\mathrm{i} f\left(\partial_{y^{\prime}} \ln \bar{\eta}_{M}^{F}\left(y^{\prime}\right)\right)-f^{2}} \\
& \left.\quad-\left(f_{L} / \bar{\eta}_{M}^{F}(y)\right)\right),
\end{aligned}
$$

and the transmitted field is related to the applied field as

$$
\begin{aligned}
& \int \mathrm{d} y^{\prime} \widetilde{F}_{M}^{(\mathrm{r})}\left(0, y^{\prime}\right) \psi_{L}\left(f^{2} ; y^{\prime}\right) \times\left(\left(1 / \bar{\eta}_{L}^{F}\right)\right. \\
& \quad \times \sqrt{\omega^{2} \varepsilon_{M}\left(y^{\prime}\right) \mu_{M}\left(y^{\prime}\right)-\mathrm{i} f\left(\partial_{y^{\prime}} \ln \bar{\eta}_{M}^{F}\left(y^{\prime}\right)\right)-f^{2}} \\
&\left.\quad+\left(f_{L} / \bar{\eta}_{M}^{F}(y)\right)\right) \\
&=\left(2 / \bar{\eta}_{L}^{F}\right) \int \mathrm{d} y^{\prime} \widetilde{F}_{L}^{(\mathrm{r})}\left(0, y^{\prime}\right) \psi_{L}\left(f^{2} ; y^{\prime}\right) \\
& \quad \times \sqrt{\omega^{2} \varepsilon_{M}\left(y^{\prime}\right) \mu_{M}\left(y^{\prime}\right)-\mathrm{i} f\left(\partial_{y^{\prime}} \ln \bar{\eta}_{M}^{F}\left(y^{\prime}\right)\right)-f^{2}} .
\end{aligned}
$$

With piecewise constant response functions (see Figure 4),

$$
\eta_{M}^{F}(y)=\eta_{j}^{F} \quad \text { for } \quad y_{j-1}<y \leq y_{j},
$$

Eq. (60) gives

$$
\begin{aligned}
& \sum_{j=1}^{4}\left(\frac{f_{j}}{\bar{\eta}_{L}^{F}}+\frac{f_{L}}{\bar{\eta}_{j}^{F}}\right) \int_{y_{j-1}}^{y_{j}} \mathrm{~d} y^{\prime} \widetilde{F}_{L}^{(1)}\left(0, y^{\prime}\right) \psi_{L}\left(f^{2} ; y^{\prime}\right) \\
= & \sum_{j=1}^{4}\left(\frac{f_{j}}{\bar{\eta}_{L}^{F}}-\frac{f_{L}}{\bar{\eta}_{j}^{F}}\right) \int_{y_{j-1}}^{y_{j}} \mathrm{~d} y^{\prime} \widetilde{F}_{L}^{(\mathrm{r})}\left(0, y^{\prime}\right) \psi_{L}\left(f^{2} ; y^{\prime}\right) .
\end{aligned}
$$

For the following solution to the homogeneous differential equation, Eq. (14b),

$$
\begin{aligned}
& \psi_{L}\left(f^{2} ; y^{\prime}\right)=h \exp \left(\mathrm{i} f\left(y^{\prime}-1\right)\right) \\
&+h^{\prime} \exp \left(-\mathrm{i} f\left(y^{\prime}-1\right)\right),
\end{aligned}
$$

where, in order to fulfil the following boundary conditions, see Eq. (91):

$$
\begin{aligned}
\psi_{m}\left(f^{2} ; 1\right) & =h_{1}, \\
\left.\left(\partial_{y} \psi_{m}\left(f^{2} ; y\right)\right)\right|_{y=1} & =h_{2} .
\end{aligned}
$$

which leads to: (see Eq. (65)),

$$
\begin{aligned}
h & =\frac{1}{2}\left(h_{1}-\mathrm{i} h_{2} / f\right), \\
h^{\prime} & =\frac{1}{2}\left(h_{1}+\mathrm{i} h_{2} / f\right),
\end{aligned}
$$

and after multiplication with $\left(1 / h^{\prime}\right) \exp ($ if $(y-1))$, Eq. (63) becomes

$$
\begin{aligned}
& \sum_{j=1}^{4}\left(\frac{f_{j}}{\bar{\eta}_{L}^{F}}+\frac{f_{L}}{\bar{\eta}_{j}^{F}}\right) \int_{y_{j-1}}^{y_{j}} \mathrm{~d} y^{\prime} \widetilde{F}_{L}^{(1)}\left(0, y^{\prime}\right) \\
& \quad \times\left(\frac{h}{h^{\prime}} \exp \left(\mathrm{i} f\left(y+y^{\prime}-2\right)\right)+\exp \left(\mathrm{i} f\left(y-y^{\prime}\right)\right)\right) \\
& =\sum_{j=1}^{4}\left(\frac{f_{j}}{\bar{\eta}_{L}^{F}}-\frac{f_{L}}{\bar{\eta}_{j}^{F}}\right) \int_{y_{j-1}}^{y_{j}} \mathrm{~d} y^{\prime} \widetilde{F}_{L}^{(\mathrm{r})}\left(0, y^{\prime}\right) \\
& \quad \times\left(\frac{h}{h^{\prime}} \exp \left(\mathrm{i} f\left(y+y^{\prime}-2\right)\right)+\exp \left(\mathrm{i} f\left(y-y^{\prime}\right)\right)\right),
\end{aligned}
$$

When $y_{0}<y<y_{1}$, we divide Eq. (68) by $f_{1} / \bar{\eta}_{L}^{F}+f_{L} / \bar{\eta}_{1}^{F}$ and obtain

$$
\begin{aligned}
\int_{y_{0}}^{y_{1}} \mathrm{~d} y^{\prime} \widetilde{F}_{L}^{(1)}\left(0, y^{\prime}\right) \\
\quad \times\left(\frac{h}{h^{\prime}} \exp \left(\mathrm{i} f\left(y+y^{\prime}-2\right)\right)+\exp \left(\mathrm{i} f\left(y-y^{\prime}\right)\right)\right) \\
\quad+\sum_{j=2}^{4} \frac{f_{j} / \bar{\eta}_{L}^{F}+f_{L} / \bar{\eta}_{j}^{F}}{f_{1} / \bar{\eta}_{L}^{F}+f_{L} / \bar{\eta}_{1}^{F}} \int_{y_{j-1}}^{y_{j}} \mathrm{~d} y^{\prime} \widetilde{F}_{L}^{(1)}\left(0, y^{\prime}\right) \\
\quad \times\left(\frac{h}{h^{\prime}} \exp \left(\mathrm{i} f\left(y+y^{\prime}-2\right)\right)+\exp \left(\mathrm{i} f\left(y-y^{\prime}\right)\right)\right) \\
=\sum_{j=1}^{4} \frac{f_{j} / \bar{\eta}_{L}^{F}-f_{L} / \bar{\eta}_{j}^{F}}{f_{1} / \bar{\eta}_{L}^{F}+f_{L} / \bar{\eta}_{1}^{F}} \int_{y_{j-1}}^{y_{j}} \mathrm{~d} y^{\prime} \widetilde{F}_{L}^{(\mathrm{r})}\left(0, y^{\prime}\right) \\
\quad \times\left(\frac{h}{h^{\prime}} \exp \left(\mathrm{i} f\left(y+y^{\prime}-2\right)\right)+\exp \left(\mathrm{i} f\left(y-y^{\prime}\right)\right)\right) .
\end{aligned}
$$

Integration of this equation over $f$ from minus to plus infinity gives

$$
\widetilde{F}_{L 1}^{(1)}(0, y)=\int \mathrm{d} f \widehat{F}_{L 1}^{(1)}\left(f^{2}\right) \exp (\mathrm{i} f y), \quad y_{0}<y<y_{1},
$$

where the integration path lies below all singularities in the complex $f$-plane and where

$$
\begin{aligned}
& \widehat{F}_{L 1}^{(1)}\left(f^{2}\right)=r_{L 1}^{F}\left(f^{2}\right) \widehat{F}_{L 1}^{(\mathrm{r})}\left(f^{2}\right), \\
& \widehat{F}_{L 1}^{(\mathrm{r})}\left(f^{2}\right)=\frac{1}{2 \pi} \int_{y_{0}}^{y_{1}} \mathrm{~d} y^{\prime} \widetilde{F}_{L}^{(\mathrm{r})}\left(0, y^{\prime}\right) \exp \left(-\mathrm{i} f y^{\prime}\right),
\end{aligned}
$$


with

$$
r_{L j}^{F}=\frac{f_{j} / \bar{\eta}_{L}^{F}-f_{L} / \bar{\eta}_{j}^{F}}{f_{j} / \bar{\eta}_{L}^{F}+f_{L} / \bar{\eta}_{j}^{F}}
$$

When $y_{1}<y<y_{2}$, we divide Eq. (68) by $f_{2} / \bar{\eta}_{L}^{F}+f_{L} / \bar{\eta}_{2}^{F}$ and obtain

$$
\begin{aligned}
\sum_{j \neq 2}^{4} \frac{f_{j} / \bar{\eta}_{L}^{F}+f_{L} / \bar{\eta}_{j}^{F}}{f_{2} / \bar{\eta}_{L}^{F}+f_{L} / \bar{\eta}_{2}^{F}} \int_{y_{j-1}}^{y_{j}} \mathrm{~d} y^{\prime} \widetilde{F}_{L}^{(1)}\left(0, y^{\prime}\right) \\
\quad \times\left(\frac{h}{h^{\prime}} \exp \left(\mathrm{i} f\left(y+y^{\prime}-2\right)\right)+\exp \left(\mathrm{i} f\left(y-y^{\prime}\right)\right)\right) \\
\quad+\int_{y_{1}}^{y_{2}} \mathrm{~d} y^{\prime} \widetilde{F}_{L}^{(1)}\left(0, y^{\prime}\right) \\
\quad \times\left(\frac{h}{h^{\prime}} \exp \left(\mathrm{i} f\left(y+y^{\prime}-2\right)\right)+\exp \left(\mathrm{i} f\left(y-y^{\prime}\right)\right)\right) \\
=\sum_{j=1}^{4} \frac{f_{j} / \bar{\eta}_{L}^{F}-f_{L} / \bar{\eta}_{j}^{F}}{f_{2} / \bar{\eta}_{L}^{F}+f_{L} / \bar{\eta}_{2}^{F}} \int_{y_{j-1}}^{y_{j}} \mathrm{~d} y^{\prime} \widetilde{F}_{L}^{(\mathrm{r})}\left(0, y^{\prime}\right) \\
\quad \times\left(\frac{h}{h^{\prime}} \exp \left(\mathrm{i} f\left(y+y^{\prime}-2\right)\right)+\exp \left(\mathrm{i} f\left(y-y^{\prime}\right)\right)\right) .
\end{aligned}
$$

Integration of this equation over $f$ from minus to plus infinity now yields for $y_{1}<y<y_{2}$ that

$$
\begin{aligned}
\widetilde{F}_{L 2}^{(1)}(0, y) & +\int \mathrm{d} f \frac{f_{1} / \bar{\eta}_{L}^{F}+f_{L} / \bar{\eta}_{1}^{F}}{f_{2} / \bar{\eta}_{L}^{F}+f_{L} / \bar{\eta}_{2}^{F}} \widehat{F}_{L 1}^{(1)}\left(f^{2}\right) \exp (\mathrm{i} f y) \\
& =\int \mathrm{d} f \frac{f_{2} / \bar{\eta}_{L}^{F}-f_{L} / \bar{\eta}_{2}^{F}}{f_{2} / \bar{\eta}_{L}^{F}+f_{L} / \bar{\eta}_{2}^{F}} \widehat{F}_{L 2}^{(\mathrm{r})}\left(f^{2}\right) \exp (\mathrm{i} f y) \\
& +\int \mathrm{d} f \frac{f_{1} / \bar{\eta}_{L}^{F}-f_{L} / \bar{\eta}_{1}^{F}}{f_{2} / \bar{\eta}_{L}^{F}+f_{L} / \bar{\eta}_{2}^{F}} \widehat{F}_{L 1}^{(\mathrm{r})}\left(f^{2}\right) \exp (\mathrm{i} f y),
\end{aligned}
$$

where the integration paths lie below all singularities in the complex $f$-plane and where

$$
\widehat{F}_{L 2}^{(\mathrm{r})}\left(f^{2}\right)=\frac{1}{2 \pi} \int_{y_{1}}^{y_{2}} \mathrm{~d} y^{\prime} \widetilde{F}_{L}^{(\mathrm{r})}\left(0, y^{\prime}\right) \exp \left(-\mathrm{i} f y^{\prime}\right) .
$$

With the use of Eq. (70), it then follows that

$$
\widetilde{F}_{\mathrm{r}, 2}(0, y)=\int \mathrm{d} f \widehat{F}_{\mathrm{r}, 2}\left(f^{2}\right) \exp (\mathrm{i} f y), \quad y_{1}<y<y_{2},
$$

where the integration path lies below all singularities in the complex $f$-plane and where

$$
\begin{aligned}
& \widehat{F}_{L 2}^{(1)}\left(f^{2}\right)=r_{L 2}^{F}\left(f^{2}\right) \widehat{F}_{L 2}^{(\mathrm{r})}\left(f^{2}\right), \\
& \widehat{F}_{L 2}^{(\mathrm{r})}\left(f^{2}\right)=\frac{1}{2 \pi} \int_{y_{1}}^{y_{2}} \mathrm{~d} y^{\prime} \widetilde{F}_{L}^{(\mathrm{r})}\left(0, y^{\prime}\right) \exp \left(-\mathrm{i} f y^{\prime}\right) .
\end{aligned}
$$

Thus we have calculated for every slab $j=1,2,3,4$ that

$$
\widetilde{F}_{L j}^{(1)}(0, y)=\int \mathrm{d} f \widehat{F}_{L j}^{(1)}\left(f^{2}\right) \exp (\mathrm{i} f y), \quad y_{j-1}<y<y_{j},
$$

where the integration path lies below all singularities in the complex $f$-plane and where

$$
\begin{aligned}
& \widehat{F}_{L j}^{(1)}\left(f^{2}\right)=r_{L j}^{F}\left(f^{2}\right) \widehat{F}_{L j}^{(\mathrm{r})}\left(f^{2}\right), \\
& \widehat{F}_{L j}^{(\mathrm{r})}\left(f^{2}\right)=\frac{1}{2 \pi} \int_{y_{j-1}}^{y_{j}} \mathrm{~d} y^{\prime} \widetilde{F}_{L}^{(\mathrm{r})}\left(0, y^{\prime}\right) \exp \left(-\mathrm{i} f y^{\prime}\right) .
\end{aligned}
$$

Analogously, it can be calculated that the transmitted field at the interface $x=0$ is given by

$$
\widetilde{F}_{M j}^{(\mathrm{r})}(0, y)=\int \mathrm{d} f \widehat{F}_{M j}^{(\mathrm{r})}\left(f^{2}\right) \exp (\mathrm{i} f y), \quad y_{j-1}<y<y_{j}
$$

where the integration path lies below all singularities in the complex $f$-plane and where

$$
\widehat{F}_{M j}^{(1)}\left(f^{2}\right)=t_{L j}^{F}\left(f^{2}\right) \widehat{F}_{L j}^{(\mathrm{r})}\left(f^{2}\right)
$$

with

$$
t_{L j}^{F}=\frac{2 f_{j} / \bar{\eta}_{L}^{F}}{f_{j} / \bar{\eta}_{L}^{F}+f_{L} / \bar{\eta}_{j}^{F}} .
$$

A monochromatic field that propagates under an angle $\theta$ with the horizontal axis generates at the boundary interface $x=0$ the field distribution

$$
\widehat{F}_{L}^{(\mathrm{r})}(\omega ; 0, y)=\operatorname{Re}\left\{\exp \left[\mathrm{i} k_{L} y \sin \theta\right]\right\},
$$

Eq. (82) gives

$$
\begin{aligned}
\widehat{F}_{L j}^{(\mathrm{r})}\left(f^{2}\right)=\frac{1}{4 \pi \mathrm{i}} & {\left[\frac{\exp \left(\mathrm{i}\left(k_{L} \sin \theta-f\right) y_{j}\right)}{k_{L} \sin \theta-f}\right.} \\
& \left.-\frac{\exp \left(\mathrm{i}\left(k_{L} \sin \theta-f\right) y_{j-1}\right)}{k_{L} \sin \theta-f}\right] \\
& -\frac{1}{4 \pi \mathrm{i}}\left[\frac{\exp \left(-\mathrm{i}\left(k_{L}^{\star} \sin \theta+f\right) y_{j}\right)}{k_{L}^{\star} \sin \theta+f}\right. \\
& \left.-\frac{\exp \left(-\mathrm{i}\left(k_{L}^{\star} \sin \theta+f\right) y_{j-1}\right)}{k_{L}^{\star} \sin \theta+f}\right] .
\end{aligned}
$$

\section{NUMERICAL RESULTS}

The analytical results obtained above will now be numerically evaluated for the following geometry. At $x<0$, there is a vacuum. At $x>0$, we have a dielectric medium that consists of two horizontal layers in between another dielectric medium. The dielectric functions $\varepsilon_{j}(\omega), j=1 \cdots 4$ for the various layers, read as

$$
\varepsilon_{j}(\omega)=\varepsilon_{0}+\frac{\varepsilon_{0} \omega_{p j}^{2}}{\omega_{j}^{2}-\omega^{2}-2 \mathrm{i} \gamma_{j} \omega} \quad j=1, \ldots, 4 .
$$

The numerical values of the parameters in Eq. (88) are given in Table 1. Note that the first and fourth layer are given identical optical properties. The medium is illuminated by

\begin{tabular}{|c|c|c|c|}
\hline \multicolumn{4}{|c|}{ Slab parameter values } \\
\hline & $l_{2}=200 \mathrm{~nm}$ & $l_{3}=300 \mathrm{~nm}$ & \\
\hline$\omega_{p 1}=1.5$ & $\omega_{p 2}=4.5$ & $\omega_{p 3}=2.0$ & $\omega_{p 4}=1.5$ \\
\hline$\omega_{1}=4.0$ & $\omega_{2}=4.0$ & $\omega_{3}=2.5$ & $\omega_{4}=4.0$ \\
\hline$\gamma_{1}=0.10$ & $\gamma_{2}=0.20$ & $\gamma_{3}=0.15$ & $\gamma_{4}=0.10$ \\
\hline
\end{tabular}

TABLE 1 Slab parameter values, where frequencies are in units of $10^{16} \mathrm{rad} / \mathrm{s}$. All media have $\mu_{j}=\mu_{0}$

the monochromatic incoming field specified above, where we take for the angular frequency $\omega_{0}=4.0 \cdot 10^{15} \mathrm{rad} / \mathrm{s}$ and for the angle $\theta=\frac{\pi}{8}$. The field distributions at $x=0$ of this incoming field and the resulting transmitted and reflected fields that were calculated with the above formulae have been plotted in Figure 5. 


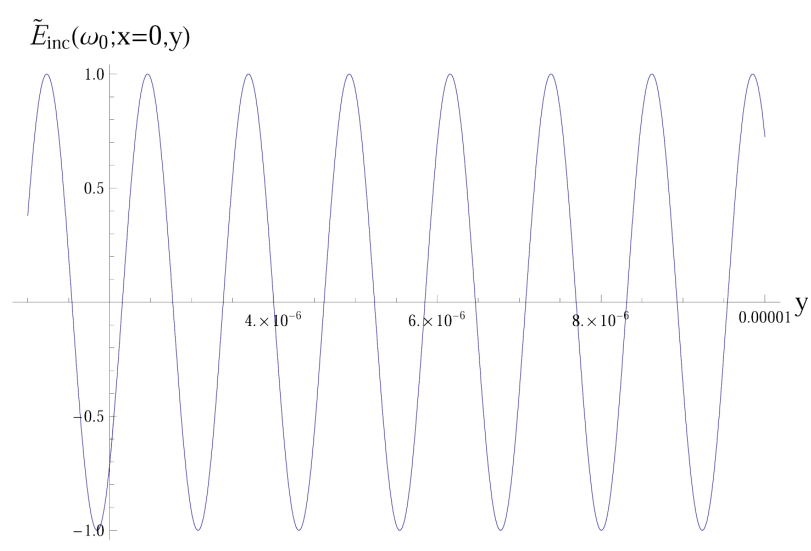

$\tilde{E}_{\mathrm{tr}}\left(\omega_{0} ; \mathrm{x}=0, \mathrm{y}\right)$

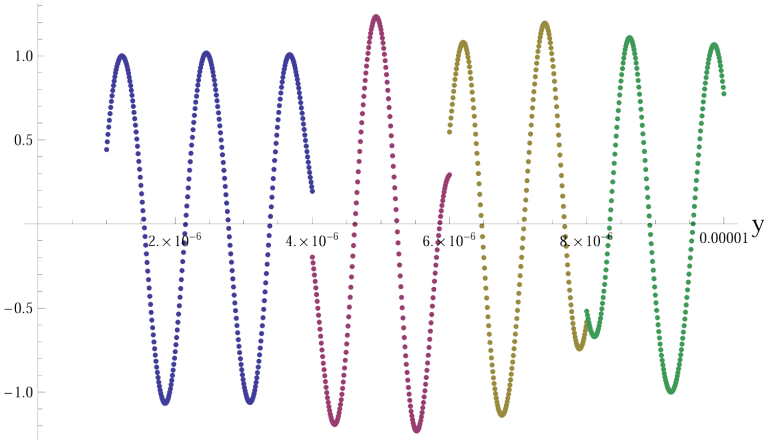

$\tilde{E}_{\text {ref }}\left(\omega_{0} ; \mathrm{x}=0, \mathrm{y}\right)$

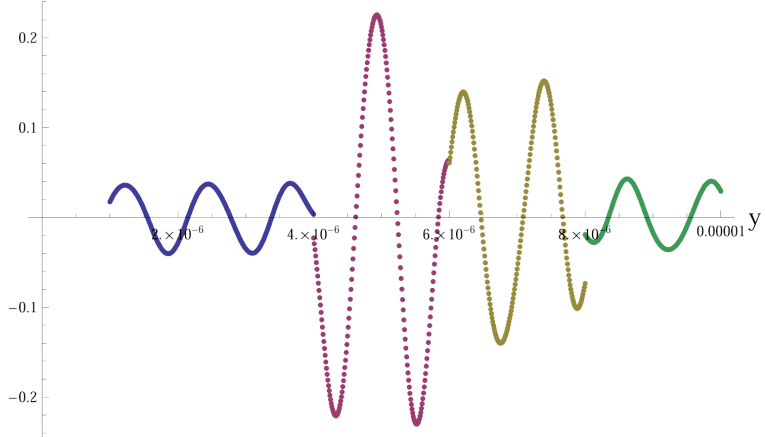

FIG. 5 Distributions of the incoming, transmitted and reflected fields at the interface at $x=0$ between a homogeneous medium to the left and a horizontal set of two layers to the right.

\section{DISCUSSION}

The usual theory for the solution of boundary value problems is based on the theory of eigenfunction expansions for separable coordinate systems for the pertinent scalar- or vectorial wave equations. Then, for geometries of the scattering medium coinciding with these separable coordinate systems, the scattering problem can be solved in terms of the appropriate eigenfunctions [1, 2]. If, however, the boundaries of the scatterer do not coincide fully with a separable coordinate system, this theory is not applicable but the theory developed above gives the possibility to perform an exact calculation. An example of such an extension of the usual theory is provided by the scattering of a wave from the configuration drawn in Figure 2. Moreover, we would like to observe that scattering by a multilayer slab whose layers are neither parallel- or perpendicular to the boundary planes of the slab can be exactly calculated with our theory if a new coordinate system is introduced such that the $y$-axis stays as shown in Figure 2, whereas the $x$-axis is parallel with the layers. Another example of the extension of the usual theory made possible by our new theory of eigenfunction expansions is that of the scattering by, for instance, slabs whose optical properties are changing in both the $x$ and $y$ directions, provided the pertinent wave equations separate. So, e.g., scattering by a square structure consisting of $N$ layers of constant refractive index $n_{1}$ in the $x$-direction superposed on $M$ layers with constant index of refraction $n_{2}$ in the $y$-direction can be exactly calculated. The theory developed in this paper can also be generalized for the analysis of the scattering properties of a structure consisting of regularly placed pyramids. Such a structure occurs e.g. at the cornea of moths, [11]. Another example of a now solvable scattering problem using the methods of this paper would be that of a slab with sinusoidal changing refractive in$\operatorname{dex} n^{2}(x, y)=A \sin (x)+B \sin (y)$, where $A$ and $B$ denote constants. The field modes are then the Mathieu functions.

The key feature of the theory, put forward by us, is the introduction of a new set of modes described in App. 7. They can be interpreted as the modes generated by a driven wire or membrane with a "driving force" $K_{M}^{F}\left(f^{2} ; y\right)$. The key property of these modes is that the completeness relation is in terms of these modes and a related set of modes of the homogeneous equation, i.e. free space modes, see Eq. (105). This enables the expansion of the fields into either set of modes. This essential property of the modes then leads to an analysis of the continuity conditions quite similar to those in case of scattering of an incoming wave by a homogeneous medium. The involved pertinent spectral densities satisfy a linear set of equations. These equations are very similar compared to the ones obtained in case of scattering by a slab of homogeneous material, in which case the spectral densities are connected with the various expansions of the fields into plane waves.

At first sight the numerical implementation of this theory might seem to be rather difficult. The expansions depend e.g. on the calculation of the roots of a transcendental equation, Eq. (98), whereafter then several infinite summations have to be evaluated. However, all these summations are expressed in terms of closed analytical formulae, viz. the contour integral of Eq. (104) of App. 7, and can thus be calculated evaluating an integral with explicitly known argument.

\section{DERIVATION OF HYBRID COMPLETENESS RELATIONS}

In this appendix we derive the various theorems pertinent to the series expansion of Eqs. (18) which is fundamental for the theory developed in this paper. Consider the solutions $\psi_{L}^{(1)}\left(f^{2} ; y\right)$ and $\psi_{L}^{(2)}\left(f^{2} ; y\right)$ of the homogeneous differential equation

$$
\left(\partial_{y}^{2}+f^{2}\right) \psi_{m}\left(f^{2} ; y\right)=0
$$


that satisfy

$$
\begin{aligned}
\psi_{m}^{(1)}\left(f^{2} ; 1\right) & =1, \\
\left.\left(\partial_{y} \psi_{m}^{(1)}\left(f^{2} ; y\right)\right)\right|_{y=1} & =0, \\
\psi_{m}^{(2)}\left(f^{2} ; 1\right) & =0, \\
\left.\left(\partial_{y} \psi_{m}^{(2)}\left(f^{2} ; y\right)\right)\right|_{y=1} & =1 .
\end{aligned}
$$

The above solutions are independent, because their Wronskian equals one. The general solution to Eq. (89) reads as

$$
\psi_{m}\left(f^{2} ; y\right)=h_{1} \psi_{m}^{(1)}\left(f^{2} ; y\right)+h_{2} \psi_{m}^{(2)}\left(f^{2} ; y\right),
$$

where $h_{1}$ and $h_{2}$ are constants. The solutions to the inhomogeneous differential equation,

$$
\left(\partial_{y}^{2}+f^{2}\right) \psi_{M}\left(f^{2} ; y\right)=K_{M}^{F}\left(f^{2} ; y\right),
$$

can be constructed with the help of the function

$$
\begin{aligned}
\psi_{L}^{(4)}\left(f^{2} ; y, y^{\prime}\right)= & \psi_{L}^{(1)}\left(f^{2} ; y\right) \psi_{L}^{(2)}\left(f^{2} ; y^{\prime}\right) \\
& -\psi_{L}^{(2)}\left(f^{2} ; y\right) \psi_{L}^{(1)}\left(f^{2} ; y^{\prime}\right)
\end{aligned}
$$

as

$$
\begin{aligned}
\psi_{M}\left(f^{2} ; y\right)= & \int_{0}^{y} \mathrm{~d} y^{\prime} K_{M}^{F}\left(f^{2} ; y^{\prime}\right) \\
& \times \psi_{L}^{(4)}\left(f^{2} ; y^{\prime}, y\right)+\bar{\psi}_{L}\left(f^{2} ; y\right),
\end{aligned}
$$

where

$$
\begin{aligned}
\bar{\psi}_{L}\left(f^{2} ; y\right)= & \beta \psi_{m}^{(4)}\left(f^{2} ; 0, y\right) \\
& -\left.\alpha\left(\partial_{y^{\prime}} \psi_{m}^{(4)}\left(f^{2} ; y^{\prime}, y\right)\right)\right|_{y^{\prime}=0}
\end{aligned}
$$

in which the arbitrary constants $\alpha$ and $\beta$ fix the boundary conditions of $\psi_{M}$ at $y=0$ as

$$
\begin{array}{r}
\psi_{M}\left(f^{2} ; y=0\right)=\alpha, \\
\left.\left(\partial_{y} \psi_{M}\left(f^{2} ; y\right)\right)\right|_{y=0}=\beta,
\end{array}
$$

and where it is supposed that the function $K_{M}^{F}$ is integrable on the interval $0 \leq y \leq 1$. It can be verified, that

$$
\left.h_{1}\left(\partial_{y} \psi_{M}\left(f^{2} ; y\right)\right)\right|_{y=1}-h_{2} \psi_{M}\left(f^{2} ; 1\right)=\gamma+N\left(f^{2}\right) \text {, }
$$

where $\gamma$ is a constant and

$$
\begin{aligned}
N\left(f^{2}\right)= & \int_{0}^{1} \mathrm{~d} y \psi_{m}\left(f^{2} ; y\right) K_{M}^{F}\left(f^{2} ; y\right) \\
& -\gamma+\beta \psi_{m}\left(f^{2} ; 0\right)-\left.\alpha\left(\partial_{y} \psi_{m}\left(f^{2} ; y\right)\right)\right|_{y=0}
\end{aligned}
$$

is a discretisation condition for the separation variable $f^{2}$. Let $G$ denote the Green function that satisfies the differential equation

$$
\left(\partial_{y}^{2}+f^{2}\right) G\left(f^{2} ; y, y^{\prime}\right)=\delta\left(y-y^{\prime}\right),
$$

the boundary conditions

$$
\begin{aligned}
G\left(f^{2} ; 1, y^{\prime}\right) & =h_{1} \rho\left(f^{2} ; y^{\prime}\right), \\
\left.\partial_{y} G\left(f^{2} ; y, y^{\prime}\right)\right|_{y=1} & =h_{2} \rho\left(f^{2} ; y^{\prime}\right),
\end{aligned}
$$

with $\rho$ a function to be determined, and the eigenvalue equation

$$
\begin{aligned}
\int_{0}^{1} \mathrm{~d} y^{\prime} G & \left(f^{2} ; y^{\prime}, y\right) K_{M}^{F}\left(f^{2} ; y^{\prime}\right) \\
& -\rho\left(f^{2} ; y\right) \gamma+\beta G\left(f^{2} ; 0, y\right) \\
& -\left.\alpha\left(\partial_{y^{\prime}} G\left(f^{2} ; y^{\prime}, y\right)\right)\right|_{y^{\prime}=0}=0 .
\end{aligned}
$$

From Eqs. (99) and (100), it follows that

$$
\begin{aligned}
& G\left(f^{2} ; y, y^{\prime}\right) \\
& \quad= \begin{cases}\psi_{m}\left(f^{2} ; y\right) \rho\left(f^{2} ; y^{\prime}\right) & \text { if } y \geq y^{\prime}, \\
\psi_{m}\left(f^{2} ; y\right) \rho\left(f^{2} ; y^{\prime}\right)+\psi_{m}^{(4)}\left(f^{2} ; y, y^{\prime}\right) & \text { if } y \leq y^{\prime} .\end{cases}
\end{aligned}
$$

Eq. (101) then gives

$$
\rho\left(f^{2} ; y\right)=\frac{\psi_{M}\left(f^{2} ; y\right)}{N\left(f^{2}\right)} .
$$

From the theory of residues and from Eqs. (102) and (103), it follows that

$$
\begin{aligned}
& \frac{1}{2 \pi \mathrm{i}} \oint_{\left|\lambda^{2}\right|=\Lambda^{2}} \mathrm{~d} \lambda^{2} \frac{G\left(\lambda^{2} ; y, y^{\prime}\right)}{\lambda^{2}-f^{2}} \\
& \quad=G\left(f^{2} ; y, y^{\prime}\right)+\sum_{n} \frac{\psi_{m}\left(f_{n}^{2} ; y\right) \psi_{M}\left(f_{n}^{2} ; y^{\prime}\right)}{\left(f_{n}^{2}-f^{2}\right) N^{\prime}\left(f_{n}^{2}\right)},
\end{aligned}
$$

where $\Lambda^{2}$ denotes a very large constant, where

$$
N^{\prime}\left(f_{n}^{2}\right)=\left.\left(\mathrm{d} N / \mathrm{d} f^{2}\right)\right|_{f^{2}=f_{n}^{2}}
$$

and where the $f_{n}^{2}$ are the solutions of the transcendental equation (98), viz. $N\left(f_{n}^{2}\right)=0$. In the limit $\Lambda^{2} \rightarrow \infty$, the integral in Eq. (104) vanishes. From applying $\left(\partial_{y}^{2}+f^{2}\right)$ to Eq. (104), one finds the hybrid mode expansion

$$
\delta\left(y-y^{\prime}\right)=\sum_{n} \frac{\psi_{m}\left(f_{n}^{2} ; y\right) \psi_{M}\left(f_{n}^{2} ; y^{\prime}\right)}{N^{\prime}\left(f_{n}^{2}\right)}
$$

\section{References}

[1] P. M. Morse, and H. Feshbach, Methods of Theoretical Physics (McGraw-Hill, New York, 1953).

[2] V. D. Kupradze, Randwertaufgaben der Schwingungstheorie und Integralgleichungen (Deutscher Verlag der Wissenschaften, Berlin, 1956).

[3] P. H. Moon, and D. E. Spencer, Field theory handbook: including coordinate systems, differential equations and their solutions (Springer-Verlag, Berlin, 1988).

[4] S. S. S. Vinogradov, P. Smith, and E. D. Vinogradova, Canonical Problems in Scattering and Potential Theory, Part 2; Acoustic and Electromagnetic Diffraction by Canonical Structures (vol. 127 of Monographs and Surveys in Pure and Applied Mathematics, Chapman Hall, London, 1981).

[5] M. Born, and E. Wolf, Principles of Optics (Seventh Edition, Springer-Verlag, Berlin, 1999).

[6] B. J. Hoenders, M. Bertolotti, and R. Uitham, "Set of modes for the description of wave propagation through slabs with a transverse variation of the refractive index" J. Opt. Soc. Am. A 24, 1189-1200 (2007). 
[7] E. Hilb, “Über Reihenentwicklungen, welche aus speziellen Randwertproblemen bei gewönlichen linearen inhomogenen Differentialgleichungen entspringen" Journ. reine. angew. Math. 140, 205-229 (1911).

[8] B. J. Hoenders, and M. Bertolotti, "Coherence theory of electromagnetic wave propagation through stratified $\mathrm{N}$-layer media" J. Opt. Soc. Am. A 22, 1143-1150 (2005).

[9] P. Yeh, Optical Waves in Layered Media (Wiley series in pure and applied optics, John Wiley and Sons, Chicester, 1988).
[10] P. Yeh, A. Yariv, and C.-S. Hong, "Electromagnetic propagation in periodic stratified media. I. General theory" J. 0pt. Soc. Am. 67, 423-438 (1977).

[11] D. G. Stavenga, S. Foletti, G. Palasantzas, and K. Arikawa, "Light on the moth-eye corneal nipple array of butterflies" Proc. R. Soc. B 273, 661-667 (2006). 